\title{
Eva Valesky im Gespräch mit Prof. Dr. Wolfgang Hach
}

\section{Wie kam es, dass Sie neben der Chirurgie zusätzlich das Fach Phlebologie gewählt haben?}

Mit „Phlebologie“ begann anno 1954 mein erster Arbeitstag in der Allgemein- und Unfallchirurgie in Berlin. Unter meinem Chef Dr. Schäfer haben wir alle einschlägigen Operationen der Venen und des Ulcus cruris durchgeführt, und zwar täglich bei schwerstbetroffenen Patienten. Bald hatte ich hierzu schon als junger Arzt eine beträchtliche Operationsliste.

Nach der chirurgischen Fachausbildung wollte ich in die Herzchirurgie wechseln, aber ich erhielt keine Stelle. Deshalb habe ich den Umweg über die Kardiologie in der I. Medizinischen Universitätsklinik Frankfurt versucht. Dort befand sich das Herzkatheterlabor in den Kellerräumen der Röntgenabteilung. Wir haben zu dieser Zeit vor allem die Auszugskurven der fortlaufenden Druckmessung bei der Mitralstenose und Aortenstenose erstellt. Am Nebentisch im selben Raum arbeitete der berühmte Kinderkardiologe Dr. Vetter, der jetzt gerade zu seinem 100. Geburtstag hoch geehrt wurde.

Im Röntgen-Keller der I. Medizinischen Klinik erfolgten auch die verschiedenen selektiven und superselektiven Organ-Angiografien, die ich - mit Ausnahme der zerebralen Angiografie - fast alle selbst gemacht habe. Hinzu kamen die peripheren Arteriografien. Dazu musste ich vorher jeden Patienten persönlich untersuchen, und es entstand so etwas wie eine „Angiologie“ an der Uni. Mittlerweile hatte ich den Facharzt für Innere Medizin und für Röntgendiagnostik erworben. Ein erneuter Anlauf, in die neu eingerichtete Herzchirurgie hier in Frankfurt unter Prof. Hirsch zu wechseln, hat wieder nicht geklappt.

Eines Tages meinte mein Röntgenchef Prof. Gebauer, er habe eine Arbeit über „Phlebografie“ gelesen und ich solle doch nach Innsbruck reisen, um das Verfahren in unserer Klinik einzuführen. Wir haben die venöse Phase der arteriellen Angiografie damals für die Tumordiagnostik verwendet. Es war weder von Varizen noch von Thrombosen noch von Lungenembolien die Rede an der Klinik. Prof. Gebauer muss wohl „Phlebografie“ irgendwie falsch verstanden haben. Es passte gar nicht zu uns, denn in Innsbruck bei May und Nissl ging es nur um Varizen, Thrombose und postthrombotisches Syndrom an den Bein- und Beckenvenen. Aber es war für mich eine neue, faszinierende Welt, die ich da sah, allerdings an Uralt-Röntgengeräten. In Frankfurt habe ich alles sofort nachgemacht, jedoch stand mir hier die modernste Technik mit Röntgen-Fernsehkette usw. zur Verfügung. Sofort konnte ich mit meiner kardiovaskulären Vorbildung eine ganze Reihe von neuen Erkenntnissen gewinnen und - wie man es an der Uni macht - publizieren. Außer den arteriellen Durchblutungsstörungen bestand jetzt auch ein Interesse an phlebologischen Patienten in unserer Inneren Medizin. Gleichzeitig entwickelte mein Freund Prof. Klaus Breddin die Hämostaseologie in seinem speziellen Gerinnungslabor. $\mathrm{Er}$ war an Thrombosen interessiert. In dieser Abteilung der I. Medizinischen Universitätsklinik arbeitete sich dann später auch meine Tochter Viola ein, machte den Facharzt für Innere Medizin/Angiologie und habilitierte sich mit einem ThromboseThema.

Als ich 1969 die Frankfurter Universität verließ, um eine Chefarztstelle mit 100 Betten im DRK-Krankenhaus am Zoo zu übernehmen, fand ich Gelegenheit, meine chirurgischen Erfahrungen bei Venenkrankheiten aus der allerersten chirurgischen Klinik anzuwenden. Mit meinem Slogan „Operieren nach dem Röntgenbild" haben sich schnell viele neue Erkenntnisse ergeben. Vom Vorsitzenden der Landesärztekammer Hessen, Prof. Dr. Rheindorf, erhielt ich die Ausnahmegenehmigung, die Facharzttitel für Chirurgie, Innere Medizin und Röntgendiagnostik anzuwenden, also der Disziplinen, die auch heute die Säulen der Angiologie und Gefäßchirurgie repräsentieren. „Meine Phlebologie" ist also in einer universitären Röntgenabteilung geboren worden, nachdem Vorkenntnisse bestanden.

\section{Wer war/waren Ihr/e Lehrer? Wer hat Sie besonders beeindruckt?}

Insbesondere waren meine beiden ersten Chefs in der Chirurgie, Dr. Schäfer und Dr. Zettel, als Lehrer ausgesprochene Glücksfälle. In der Chirurgie steht man ja am Operationstisch physisch eng zusammen, und das jeden Tag über viele Stunden, jahrein - jahraus. Ich konnte schließlich bei beiden Chefs fast jeden ihrer Gedanken voraussagen. In unserem Beruf steht das Menschliche an der ersten Stelle, wie sich Arzt und Patient begegnen. Dr. Schäfer und Dr. Zettel waren die perfekten Vorbilder. Später brauchte ich in zweifelhaften Situationen nur zu überlegen, wie hätten sie sich entschieden, und ich wusste, was zu tun ist.

Die wissenschaftliche Ausbildung verdanke ich dem Direktor der I. Medizinischen Universitätsklinik Prof. Hoff, dem Oberarzt Prof. Heintz und dem Radiologen Prof. Gebauer. Später in der praktizierten Phlebologie hatte ich größte Hochachtung vor Robert May in Innsbruck und Karl Sigg in Basel.

Gibt es einen Fall aus der Phlebologie, der Ihnen besonders im Gedächtnis geblieben ist?

Ja! Ich habe die ungewöhnliche Kasuistik in unserem Buch verewigt: VenenChirurgie, 3. Aufl. S. 366. Schattauer, Stuttgart 2013. Es handelt sich um einen damals 51-jährigen Mann mit postthrombotischen Manschetten-Ulzera beiderseits, die bis zu den Zehen reichten. Ich habe deshalb den linken Vorfuß amputieren müssen. Der Patient hatte ganze 11 Jahre seines Lebens in Krankenhäusern verbracht und alle bekannten Phlebologen in Europa konsultiert, vergeblich. Mit seiner übergroßen 
Verbandtasche und mit seinem freundlichen, vornehmen Auftreten kannte man ihn vielerorts. Dann wurde seine Ehe wegen der stinkenden Verbände und ständig verschmutzten Bettwäsche geschieden und das Eigenheim verkauft; er landete auf der Straße in der Drogenszene.

Eines Tages stellte sich der Patient nach 20-jähriger ambulanter Behandlung auch in unserer Klinik wieder einmal vor und wünschte fest entschlossen, dass ich beide Unterschenkel amputiere. Er wollte seinem Leben wieder einen Sinn geben. Ich schlug ihm - nach meinen langen theoretischen Überlegungen - eine neue Operationsmethode vor, die komplette krurale Fasziektomie, erst rechts, dann links. Er willigte auf der Stelle ein. 3 Monate später waren die Ulzera komplett abgeheilt. Unser Patient fand in seinen Beruf zurück und hat seine Frau ein zweites Mal geheiratet. Viele Jahre lang kam er uns noch aus Mannheim besuchen, gut zu Fuß und ohne Verbandtasche.

\section{Was ist die wichtigste Erkenntnis der vergangenen Jahre in der Phlebologie?}

Mittels der Phlebografie konnte vor 50 Jahren eine sehr genaue Diagnostik der Venenkrankheiten und demzufolge auch eine differenzierte Therapie erfolgen. Jede Perforansinsuffizienz war abgebildet, der kleinste Thrombus in einer Klappentasche erkennbar, der erste Beginn der sekundären Leitveneninsuffizienz mit Sicherheit zu analysieren und dokumentieren. Allerdings erforderte eine gute Phlebografie auch besondere Voraussetzungen bezüglich der technischen Ausstattung und der speziellen Kenntnisse des Untersuchers.

Heute ist die Phlebografie durch die Ultraschalldiagnostik verdrängt worden. Aber auch damit werden flächendeckend im ganzen Land gute und richtige Diagnosen gestellt. Die höchste Präzision ist jedoch auf der Strecke geblieben. Perforansinsuffizienzen werden an atypischer Stelle leicht übersehen, kleinere Thromben eher der hämostaseologischen Diagnostik überlassen und die sekundäre Leitveneninsuffizienz in ihrer Bedeutung nicht ausreichend wahrgenommen. Trotzdem, die Vorteile überwiegen: Alle Risiken der invasiven Phlebografie werden durch die Nicht-Invasivität der mo-

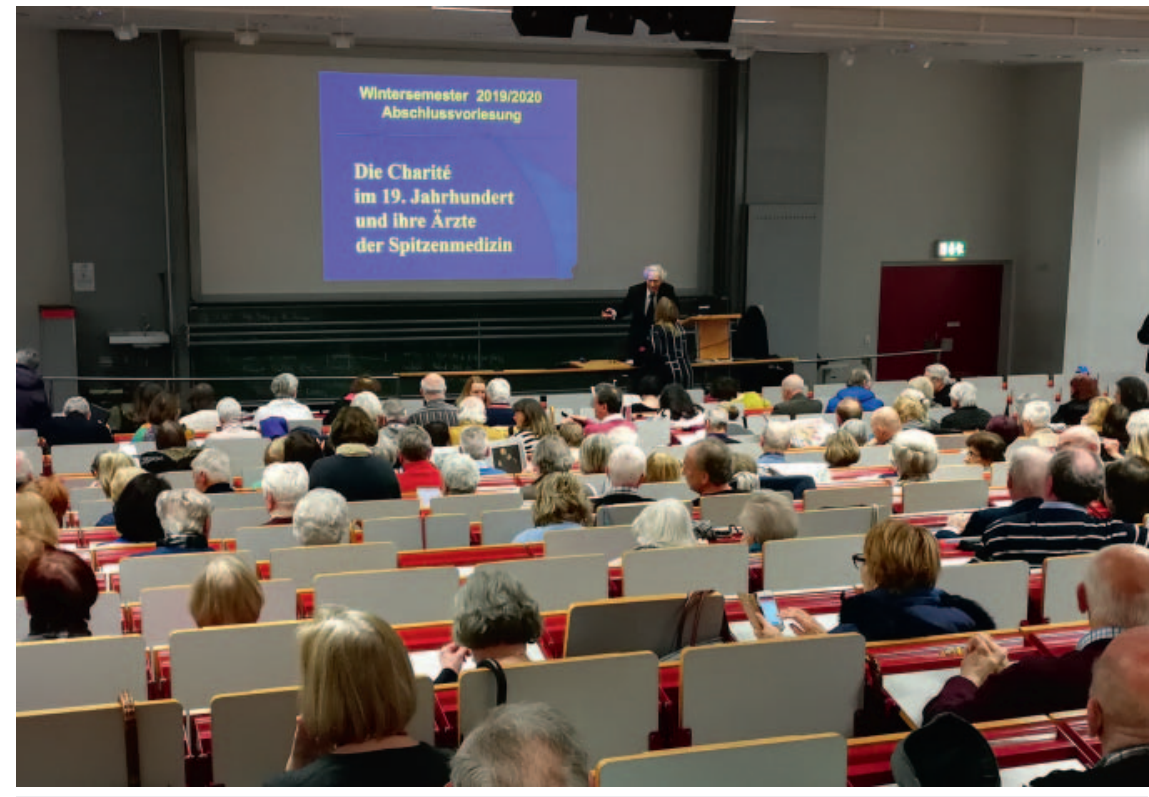

- Abb. 1 Professor Hach in seiner Abschiedsvorlesung der Universität des 3. Lebensalters im Februar 2020. Quelle: Privatarchiv Familie Hach.

dernen Sonografie eliminiert. Außerdem kann sich die Ausbildung in der Ultraschalldiagnostik für die aktuelle Phlebologie den Notwendigkeiten unserer Zeit mit ein paar Wochenendkursen anpassen. Ich habe darüber hinaus den Eindruck, dass es die sehr schweren Krankheitsverläufe von früher heute kaum noch gibt.

\section{Was ist die derzeit wegweisendste Ent- wicklung in der Phlebologie?}

In der Chirurgie der primären Varikose haben sich die verschiedenen endovenösen Methoden wegen ihrer Mini-Invasivität bewährt, und das wird auch in der Zukunft so bleiben. Trotzdem hatte ich in den letzten Jahren den Eindruck, dass die klassischen Operationsverfahren bei ausgeprägtem Krankheitsbild wiederbelebt werden. Allerdings sind viele gute Schulen der Venenchirurgie mittlerweile gestorben und mit ihnen das Know-how.

Bezüglich der Thrombose und ihrer Folgekrankheiten hat die Kompressionstherapie des Heinrich Fischer aus dem Jahr 1910 nichts an Bedeutung verloren, jedoch erleichtern neue Materialien und Techniken den Umgang damit im täglichen Leben.

Bezüglich des Ulcus cruris haben die modernen Wundauflagen die alten lokalen
Prinzipien verdrängt. Ein entscheidender Fortschritt ist mit Einführung der Vakuumversiegelung gelungen. Immerhin sollte die Phlebologie alte und bewährte Operationsverfahren beim Ulkus nicht der Vergessenheit preisgeben; dazu gehören die lokale Nekrosektomie bis zur Homans'schen Operation und sogar die paratibiale Fasziotomie.

\section{Wo sehen Sie die Zukunft der akademi- schen Phlebologie? Welche relevanten Fragen sind für Sie weiterhin ungeklärt?}

Ich habe immer die Meinung vertreten, dass die akademische Phlebologie am besten im Fachgebiet der Dermatologie aufgehoben ist. Das ist auch heute meine Ansicht. Viele dermatologische Universitätskliniken halten hierfür eine spezielle Abteilung vor, in der auch die endovenösen Verfahren bei der primären Varikose zur Anwendung kommen. Das ist gut so. Aber über den Laser hinaus darf die klassische Chirurgie für einige Krankheitsfälle nicht vergessen werden. Sie liegt in den Händen der Allgemein- und Gefäßchirurgen unter ambulanten oder stationären Bedingungen. Es geht um die korrekte Krossektomie, das Stripping und die selektive Perforansdissektion.

Ich bin nach wie vor auch der Meinung, dass die Pathophysiologie des chronischen 


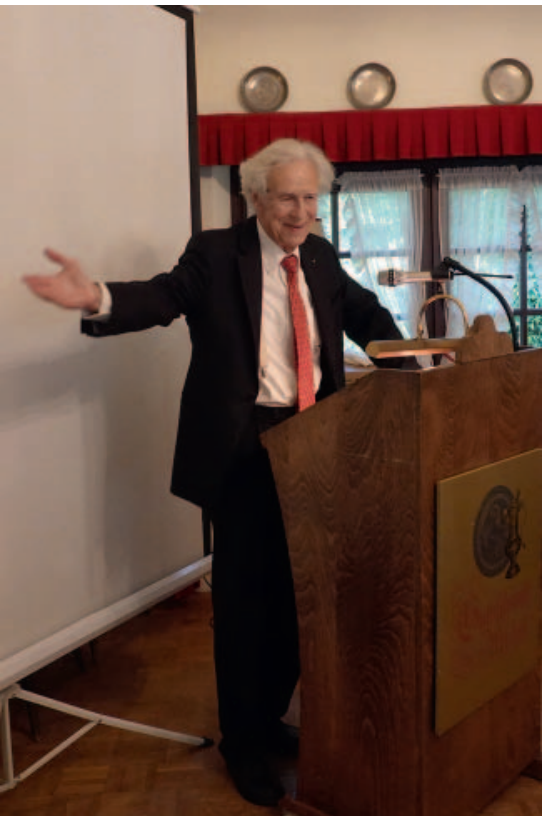

- Abb. 2 Professor Hach ist ein engagierter Redner. Quelle: Privatarchiv Familie Hach.

venösen Kompartmentsyndroms und die von uns seinerzeit inaugurierte Faszienchirurgie neu belebt werden sollten. Entsprechendes gilt für das arthrogene Stauungssyndrom. Da das Ulcus cruris ein Endpunkt dieser Krankheitszustände darstellt, erscheint diesbezüglich eine enge Zusammenarbeit zwischen Venenchirurgen und Dermatologen optimal.

Wie erklären Sie sich Ihre Liebe und Faszination zur Medizingeschichte?

Das ist eine lange Geschichte, eine Lebensgeschichte. Die Liebe zu Büchern habe ich von meiner Mutter geerbt, die zwar eine Marktfrau war, mit Büchern aber wie mit einem Baby umgegangen ist. In der Ober- schule spielte die Historie eine große Rolle, in der Deutschstunde mit der Klassik, im Englischen mit Shakespeare, im Lateinischen mit Virgil. Als wir 1958 mit der Familie vom Berliner Osten in den Westen gegangen sind, habe ich vor allem unsere Bücher gerettet.

Die entscheidende Motivation speziell für Medizingeschichte begann nach der Gründung unserer Praxis anno 1969 an der Konstabler Wache. Ganz in der Nähe befand sich das Antiquariat Hoffmann, eine Alt-Frankfurter Institution ganz im klassischen Stil mit hohen, großen Räumen, übervollen Regalen und mit diesem typischen muffigen Geruch. Hier standen unglaubliche Schätze. In der Mittagspause besuchte ich gern den Antiquar und lernte von ihm alles, was man auf diesem Gebiet so wissen muss. Es war wie ein Privatunterricht. Er zeigte mir kostbare Stücke und bereitete auch den einen oder anderen (kleinen) Kauf für den Aufbau meiner eigenen medizinischen Bibliothek vor. Außerdem besuchte ich seitdem regelmäßig die Fachauktionen, damals noch von Reiss und Auvermann in Glaskirchen/Taunus.

Normalerweise wird eine aktive Bibliothek irgendwann durch das persönliche Lebensalter begrenzt. Ich habe das große Glück, dass sich ein Enkel mit sehr guten Lateinkenntnissen und universitärer medizinischer Laufbahn dafür interessiert. Ich habe ihn von klein auf in das Medizin-Antiquariat eingeführt, er ist gewissermaßen darin aufgewachsen. Medizingeschichte ist vielerlei, Philosophie, Sprachwissenschaft, Latein, Geschichte, Kataloge, vor allem aber Ehrfurcht vor dem antiken Buch und vor seinem Autor.
Sie leben seit vielen Jahren in Frankfurt am Main. Gibt es einen Lieblingsort in der Stadt oder der Region? Welche Besonderheiten der Stadt würden Sie sich mit Ihren Gästen unbedingt ansehen?

Wir leben mit unserer großen Familie seit 1963 sehr gern in Frankfurt. Es ist eine schöne, lebhafte Stadt mit einer imposanten Historie und einer herrlichen Umgebung. Persönlich bin ich am meisten an den Kunstausstellungen im Städel und im Schirn interessiert. Früher gehörten wir zu den fleißigsten Besuchern von Oper und Theater. Unser Kongress der Deutschen Gesellschaft für Phlebologie 1981 (damals mit 2000 Teilnehmern) hatte den Neubau der berühmten Alte Oper Frankfurt nach der Zerstörung im Zweiten Weltkrieg eröffnet. Die Familie besucht gern und oft den Frankfurter Zoo; einer der Tiger gehört sogar als Pate zur Familie.

Lieber Herr Professor Hach, herzlichen Dank für Ihre Zeit!

\section{Korrespondenz:}

PD Dr. Eva Valesky

Universitätsklinikum Frankfurt

Theodor-Stern-Kai 7

60596 Frankfurt am Main

E-Mail: Eva.Valesky@kgu.de

\section{Bibliografie}

DOI https://doi.org/10.1055/a-1154-3257 Phlebologie 2020; 49: 130-132

(c) Georg Thieme Verlag KG, Stuttgart · New York ISSN 0939-978X 